\title{
Retraction Note to: MicroRNA-216b is Down-Regulated in Human Gastric Adenocarcinoma and Inhibits Proliferation and Cell Cycle Progression by Targeting Oncogene HDAC8
}

\author{
Ying Wang ${ }^{1} \cdot \mathrm{Po} \mathrm{Xu}^{2} \cdot \mathrm{Jun} \mathrm{Yao}^{1} \cdot \mathrm{Ruina} \mathrm{Yang}^{1} \cdot \mathrm{Zhenguo} \mathrm{Shi}^{2} \cdot \mathrm{Xiaojuan} \mathrm{Zhu}^{1} \cdot$ \\ Xiaoshan Feng ${ }^{1} \cdot$ Shegan Gao ${ }^{1}$
}

Published online: 5 December 2016

(C) Springer International Publishing Switzerland 2016

Retraction note to: Targ Oncol (2016) 11:197-207

DOI 10.1007/s11523-015-0390-9

This article has been retracted by agreement of the corresponding author, the First Affiliated Hospital of Henan University of Science and Technology, Luoyang, China, and the Editor-in-Chief. Errors were identified in some of the published figures, and the authors could not provide a satisfactory explanation owing to loss of the primary data.

The online version of the original article can be found at http://dx.doi. org/10.1007/s11523-015-0390-9.

Ying Wang

yingw_215@163.com

Xiaoshan Feng

1471511798@qq.com

Shegan Gao

949898789@qq.com

1 Oncology Department of the First Affiliated Hospital of Henan,

University of Science and Technology, No. 24 Jinghua Road,

Luoyang, Henan 471003, China

2 Urology Surgery Department of the First Affiliated Hospital of Henan University of Science and Technology, No. 24 Jinghua Road, Luoyang, Henan 471003, China 